\title{
Publisher's Note: Deflection and trapping of a counter-rotating vortex pair by a flat plate [Phys. Rev. Fluids 2, 124702 (2017)]
}

Monika Nitsche

(Q) (Received 19 January 2018; published 31 January 2018)

DOI: 10.1103/PhysRevFluids.3.019902

This paper was published online on 28 December 2017 with an incorrect Figure 8 and a mismatch of figure discussion and image location for Figs. 4-7, 10, and 11. Figure 8 has been replaced and the figure layout has been corrected as of 26 January 2018. 